Baleares. Ed. Mundiprensa. Madrid.

MARTÍNEZ PARRAS, J. M., M. PEINADO y F. ALCARAZ - 1985- Sobre la vegetación termófila de la cuenca mediterránea de Granada y sus áreas limítrofes. Lazaroa 8:251-268.

MOTA, J. F., J. PEÑAS, H. CASTRO, J. CABELLO \& J. S. GUIRADO -1996- Agricultural development vs biodiversity conservation: the Mediterranean semiarid vegetation in El Ejido (Almería, southeastern Spain). Biodiversity and Conservation 5: 1597-1617.

NIETO CALDERA, J. M., A. V. PÉREZ LATORRE y B. CABEZUDO -1991- Biogeografía y series de vegetación de la provincia de Málaga (España). Acta Bot. Malacitana 16(2):417-436.

NIETO CALDERA, J. M., B. CABEZUDO, A. V. PÉREZ LATORRE, D. NAVAS, P. NAVAS y Y. GIL -1998- Apuntes para el estudio del Paisaje Vegetal de la provincia de Málaga. En: M. Rebollo, F. Serrano, J. M. Nieto y B. Cabezudo (coords.). Itinerarios por Espacios Naturales de la provincia de Málaga. Universidad de Málaga y Consejería de Educación (Málaga).
PEINADO, M., F. ALCARAZ y J. M. MARTÍNEZ PARRAS -1992- Vegetation of Southeastern Spain. Flora et Vegetatio Mundi. Band X. Ed. J. Cramer. Berlín-Stuttgart.

PRADOS, J., J. L. VIVERO y J. E. HERNÁNDEZ BERMEJO -2000- Maytenus senegalensis subsp. europaea. En: Blanca, G., B. Cabezudo, J. E. Hernández Bermejo, C. M. Herrera, J. Muñoz y B. Valdés. Libro Rojo de la Flora Silvestre Amenazada de Andalucía. Vol. II (Especies vulnerables). Consejería de Medio Ambiente. Sevilla.

RIVAS MARTÍNEZ, S., F. FERNÁNDEZ GONZÁLEZ, J. LOIDI, M. LOUSA y A. PENAS -2001- Syntaxonomical checklist of the vascular plant communities of Spain and Portugal to association level. Itinera Geobotanica 14:5-341.

Aceptado para su publicación en septiembre de 2001

Dirección de los autores. Dpto. Biología Vegetal. Facultad de Ciencias. Universidad de Málaga. Campus de Teatinos, s/n. 29071, Málaga.

\title{
107. LAS COMUNIDADES CON CACTÁCEAS DEL SUR DEL PERÚ. NUEVOS DATOS SOBRE LA ALIANZA CORRYOCACTION BREVISTYLI
}

\author{
Antonio GALÁN DE MERA y José GÓMEZ CARRIÓN
}

The cactus communities in Southern Peru. New data on Corryocaction brevistyli alliance.

Palabras clave. Fitosociología, Cactaceae, Perú.

Key words. Phytosociology, Cactaceae, Peru.

Las comunidades con Cactáceas en América del Sur son propias de las vertientes occidentales y valles profundos interiores de la Cordillera Andina. Los biótopos sobre los que se asientan son muy característicos por su escasa estabilidad, con ríos de fango, riadas de derrubios y desprendimientos (Strahler, 1992). Las lluvias y tormentas que se producen en los 


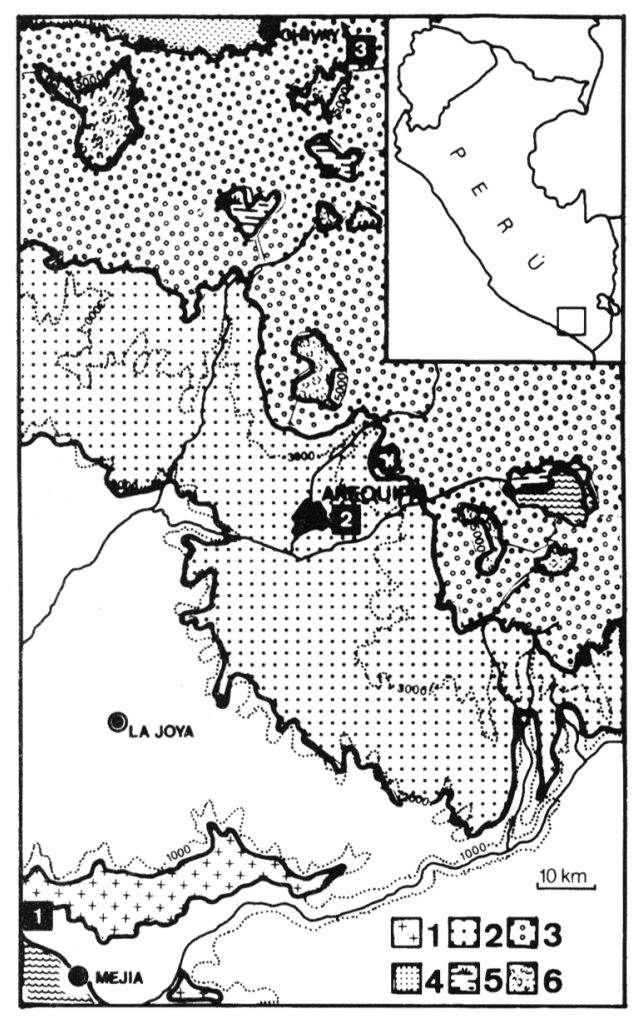

Figura 1. Mapa de los complejos de vegetación del territorio estudiado en el departamento de Arequipa (Perú). Map with the vegetation complex of the territory studied in the Arequipa Department (Peru). 1: Philoglosso peruvianae-Urocarpideto peruviani sigmetum; 2: Weberbauerocereo weberbaueriCorryocacteto brevistyli sigmetum; 3 : Parastrephio lepidophyllae sigmetalia; 4: Grindelio bolivianaeCorryocacteto puquiensis sigmetum; 5: Eleocharito tucumanensis-Plantagineto tubulosae sigmetum; 6 : Wernerio ciliolatae-Plettkeeto cryptanthae sigmetum. Los cuadrados en negro son estaciones meteorológicas. Black squares are meteorological stations: 1: Mollendo, 2: La Pampilla-Arequipa, 3: Caylloma.

Andes arrastran grandes cantidades de suelo por las laderas causando ríos de barro ( «huayco», en voz local) y, en general, pendientes poco estables, aspecto muy frecuente en áreas climáticas áridas.

Durante los trabajos de campo que hemos realizado en el sur del Perú (departamento de Arequipa), hemos levantado inventarios de vegetación (Braun-Blanquet, 1932) entre las localidades de Arequipa (2085 m) y Chivay (3554 m), con lo que, junto a los datos aportados por otros autores (Weberbauer, 1945; Aragón, 1980; Arévalo del Carpio, 1993; Linares, 2000), vamos construyendo el edificio sintax onómico de la vegetación del Perú. De esta forma, en el mapa de la figura 1 hemos cartografiado los diferentes complejos de vegetación del territorio estudiado, que según la metodología propuesta por Rivas-Martínez (1987), podemos sintetizar hasta el momento de la siguiente forma: 1: Serie termomediterránea limeñoariqueña hiperárida sabulícola de lomas Philoglosso peruvianae-Urocarpideto peruviani sigmetum; 2: Serie mesotropical oruro-arequipeña semiárido-árida acidófila de Corryocactus brevistylus- Weberbauerocereo weberbaueri-Corryocacteto brevistyli sigmetum; 3: Megaserie supra-orotropical oruro-arequipeña seca, semiárida y árida acidófila de la «tola» Parastrephia lepidophylla- Parastrephio lepidophyllae sigmetalia; 4: Serie mesotropical oruroarequipeña semiárido-árida acidófila de Corryocactus puquiensis- Grindelio bolivianae-Corryocacteto puquiensis sigmetum; 5: Serie orotropical oruroarequipeña edafohidrófila de Plantago tubulosa- Eleocharito tucumanensisPlantagineto tubulosae sigmetum; 6: Serie criorotropical andina subhúmedo-hiperhúmeda acidófila de suelos poligonales pedregososWernerio ciliolatae-Plettkeeto cryptanthae sigmetum.

En concreto, en el presente artículo, aportamos nuevos datos sobre la diversidad de la alianza Corryocaction brevistyli (OreocereoNeoraimondietalia, Opuntietea sphaericae) descrita de los Andes del sur del Perú y norte de Chile (Galán de Mera y Vicente Orellana, 1996).

La presencia en el territorio de ciertas 


\begin{tabular}{|c|c|c|c|c|}
\hline $\begin{array}{l}\text { ESTACIÓN } \\
\text { METEOROLÓGICA }\end{array}$ & ALTITUD (m) & $P$ & It & $\begin{array}{l}\text { DIAGNOSIS } \\
\text { BIOCLIMÁTICA }\end{array}$ \\
\hline $\begin{array}{l}\text { Mollendo } \\
\left(17^{\circ} 02^{\prime} \mathrm{S} / 72^{\circ} 01^{\prime} \mathrm{W}\right)\end{array}$ & 30 & 17 & 502 & $\begin{array}{c}\text { Termomediterráneo } \\
\text { hiperárido }\end{array}$ \\
\hline $\begin{array}{l}\text { Camaná } \\
\left(16^{\circ} 37^{\prime} S / 72^{\circ} 42^{\prime} \mathrm{W}\right)\end{array}$ & 40 & 15 & 513 & $\begin{array}{c}\text { Termomediterráneo } \\
\text { hiperárido }\end{array}$ \\
\hline $\begin{array}{l}\text { La Pampilla-Arequipa } \\
\left(16^{\circ} 25^{\prime} \mathrm{S} / 71^{\circ} 31^{\prime} \mathrm{W}\right)\end{array}$ & 2350 & 68 & 397 & $\begin{array}{l}\text { Mesotropical } \\
\text { árido }\end{array}$ \\
\hline $\begin{array}{l}\text { Cotahuasi } \\
\left(15^{\circ} 12^{\prime} \mathrm{S} / 72^{\circ} 54^{\prime} \mathrm{W}\right)\end{array}$ & 2683 & 247 & 441 & $\begin{array}{l}\text { Mesotropical } \\
\text { semiárido }\end{array}$ \\
\hline $\begin{array}{l}\text { Pampacolca } \\
\left(15^{\circ} 42^{\prime} \mathrm{S} / 72^{\circ} 34^{\prime} \mathrm{W}\right)\end{array}$ & 3000 & 227 & 379 & $\begin{array}{l}\text { Mesotropical } \\
\text { semiárido }\end{array}$ \\
\hline $\begin{array}{l}\text { Sibayo } \\
\left(15^{\circ} 38^{\prime} S / 71^{\circ} 27^{\prime} \mathrm{W}\right)\end{array}$ & 3810 & 562 & 78 & $\begin{array}{l}\text { Orotropical } \\
\text { subhúmedo }\end{array}$ \\
\hline $\begin{array}{l}\text { Angostura } \\
\left(15^{\circ} 11^{\prime} \mathrm{S} / 71^{\circ} 39^{\prime} \mathrm{W}\right)\end{array}$ & 4155 & 659 & 78 & $\begin{array}{l}\text { Orotropical } \\
\text { subhúmedo }\end{array}$ \\
\hline $\begin{array}{l}\text { Caylloma } \\
\left(15^{\circ} 11^{\prime} \mathrm{S} / 71^{\circ} 46^{\prime} \mathrm{W}\right)\end{array}$ & 4320 & 639 & 83 & $\begin{array}{l}\text { Orotropical } \\
\text { subhúmedo }\end{array}$ \\
\hline $\begin{array}{l}\text { Imata } \\
\left(15^{\circ} 50^{\prime} \mathrm{S} / 71^{\circ} 05^{\prime} \mathrm{W}\right)\end{array}$ & 4436 & 569 & 22 & $\begin{array}{l}\text { Criorotropical } \\
\text { subhúmedo }\end{array}$ \\
\hline
\end{tabular}

Tabla 1. Características bioclimáticas en las estaciones meteorológicas del departamento de Arequipa, Perú ( $\mathrm{P}=$ precipitación media anual, en $\mathrm{mm}$; It = Índice de termicidad de Rivas-Martínez). Bioclimatic characteristics in the meteorological stations of the Arequipa Department, Peru ( $P=$ mean annual precipitation, in $\mathrm{mm}$; $I t=$ thermicity index of Rivas Martinez).

especies de distribución óptima en el sur del Perú (además del norte de Chile y oeste de Bolivia), como Browningia candelaris, Corryocactus brevistylus, Haageocereus platinospinus, Oreocereus hempelianus, Opuntia ignescens u O. soehrensii (Brako y Zarucchi, 1993; Navarro, 1996) permite incluirlo en la provincia fitogeográfica OruroArequipeña de la superprovincia de la Puna, dentro de la región Andina (Galán de Mera, 1994).

Desde el punto de vista bioclimático, la provincia Oruro-Arequipeña disfruta de clima tropical, lo que la diferencia del clima de carácter mediterráneo de la costa del departamento de Arequipa (Galán de Mera, 2000). Según las estaciones meteorológicas y las altitudes que se encuentran dentro del territorio (fig. 1, tab. 1), los termotipos y ombrotipos oscilan entre mesotropical-criorotropical y árido-subhúmedo respectivamente, de acuerdo con la tipología de Rivas-Martínez (1997).

La nominación y tipificación de los sintáxones sigue a Barkman et al. (1986). La nomenclatura de los táxones es la de Tryon (1964) y Brako y Zarucchi (1993). 
Tabla 2

Weberbauerocereo weberbaueri-Corryocactetum brevistyli Galán de Mera y Gómez Carrión ass. nova (Corryocaction brevistyli, Oreocereo-Neoraimondietalia arequipensis, Opuntietea sphaericae)

\begin{tabular}{lccccccc}
\hline Inventario $\mathrm{n}^{\circ}$ & 1 & 2 & 3 & 4 & 5 & 6 & 7 \\
Área $^{2}$ & 100 & 100 & 100 & 100 & 100 & 100 & 100 \\
Altitud (m) & 2395 & 2800 & 2500 & 3150 & 3150 & 3270 & 2600 \\
Orientación & - & $\mathrm{S}$ & $\mathrm{SE}$ & - & - & $\mathrm{N}$ & $\mathrm{S}$ \\
Pendiente (\%) & - & 60 & 70 & - & - & 5 & 60
\end{tabular}

Características de asociación y unidades superiores

Corryocactus brevistylus

Opuntia sphaerica

Ambrosia artemisioides

Weberbauerocereus weberbaueri

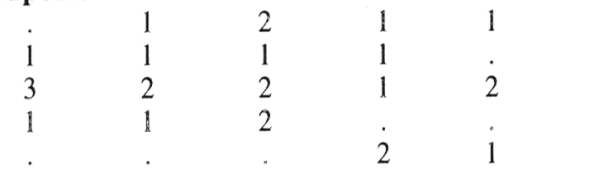

\begin{tabular}{ll}
1 & 2 \\
2 & 1 \\
. & 2 \\
& 1 \\
\hline
\end{tabular}

Características de Parastrephietalia y Calamagrostietea vicunarum

Diplostephium tacorense

Balbisia meyeniana

Bromus villosus

Echinopsis pamparuizii

Opuntia soehrensii

Stipa ichu

Compañeras

Tarasa rahmeri

Brassicaceae

Ephedra americana

Eragrostis weberbaueri

Philippiamra pachyphylla

Spergularia congestifolia

Senecio richii

Lycopersicon chilense

Muhlenbergia peruviana

Proustia berberidifolia

Tagetes multiflora

\begin{tabular}{|c|c|c|c|c|c|}
\hline • & & & 4 & 4 & 1 \\
\hline . & 1 & 1 & ; & . & . \\
\hline . & . & . & 1 & . & + \\
\hline . & . & $\cdot$ & $\cdot$ & . & + \\
\hline v & r & $\cdot$ & $i$ & . & 1 \\
\hline . & $\cdot$ & $\cdot$ & 1 & $\cdot$ & $\cdot$ \\
\hline 2 & 3 & 1 & 1 & 1 & 3 \\
\hline+ & 1 & . & 1 & + & . \\
\hline+ & . & . & . & . & + \\
\hline 4 & . & ; & 1 & . & . \\
\hline . & + & 2 & . & . & v \\
\hline . & . & . & + & & + \\
\hline · & + & & . & . & + \\
\hline · & + & + & . & . & . \\
\hline$\cdot$ &. & $\cdot$ & . &. & $\stackrel{+}{1}$ \\
\hline 2 & . & . & . & . & . \\
\hline · & . & . & . & + & . \\
\hline & + & . & . & . & \\
\hline 1 & . & . & . & . & . \\
\hline
\end{tabular}

Bryophyta

Lycium $s p$.

Encelia canescens

Localidades.- 1 a 7: Entre Arequipa y el volcán Chachani.

\section{NOVEDADES SINTAXONÓMICAS}

\section{Weberbauerocereo weberbaueri-Corryocactetum brevistyli Galán de Mera y Gómez Carrión ass. nova}

[Tabla 2, sintypus: inv. 3]

Cardonales que se asientan sobre las rocas volcánico-sedimentarias que forman las fuertes pendientes aluviales cuaternarias inestables de laderas de volcanes (Misti, Chachani, Ampato, etc.), cañones excavados sobre materiales volcánicos cuaternarios y planicies aluviales con grandes depósitos eólicos (IGM, 1975) situados entre 2000 y $3600 \mathrm{~m}$ de altitud.

La estructura de esta vegetación consiste en una cierta abundancia de caméfitos (Ambrosia artemisioides, Encelia canescens, Tarasa rahmeri), terófitos (Eragrostis weberbaueri, Spergularia congestifolia, Philippiamra pachyphylla) y cactáceas columnares dispersas (Corryocactus brevistylus, Weberbauerocereus weberbaueri) configurando el paisaje propio del bioclima mesotropical semiárido-árido del sur del Perú. Fitotopográficamente esta asociación forma una 
Tabla 3

Grindelio bolivianae-Corryocactetum puquiensis Galán de Mera y Gómez Carrión ass. nova (Corryocaction brevistyli, Oreocereo-Neoraimondietalia arequipensis, Opuntietea sphaericae)

Inventario $\mathrm{n}^{\circ}$

Área $\mathrm{m}^{2}$

Altitud (m)

Orientación

Pendiente $(\%)$

$\begin{array}{cccc}1 & 2 & 3 & 4 \\ 100 & 100 & 100 & 50 \\ 3475 & 3280 & 3735 & 3732 \\ \mathrm{NE} & \mathrm{NE} & \mathrm{NE} & \mathrm{NE} \\ 60 & 60 & 50 & 90\end{array}$

Características de asociación y unidades superiores

Corryocactus puquiensis

Grindelia boliviana

2

Opuntia sphaerica

Opuntia exaltata

Proustia oblongifolia

Corryocactus brevistylus

Características de Polypodio-Tillandsietea

Puya ferruginea

Tillandsia usneoides

Características de Notholaenetea niveae

Cheilanthes pruinata

Villadia sp.

Notholaena nivea

Pitcairnia sp.

Características de Parastrephietalia y Calamagrostietea vicunarum

Opuntia soehrensii

Diplostephium tacorense

Stipa ichu

Plantas nitrófilas y de suelos removidos

Ophryosporus peruvianus

Tarasa rahmeri

Agave americana

Conyza bonariensis

Sarcostemma solanoides

Alonsoa acutifolia

Chenopodium petiolare

Compañeras

Proustia berberidifolia

Tagetes multiflora

Ephedra americana

Eragrostis weberbaueri

Dunalia spinosa

Aristida adscensionis

Chersodoma jodoppapa

Mutisia acuminata

Senecio richii

Calamagrostis humboldtiana

Spergularia congestifolia

Nassella asplundii

Caiophora superba

Artemisia sp.

1

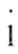

. 3

i

$+$

$+$

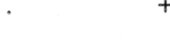

Localidades.- Departamento de Arequipa: 1: Yanque, 2: Salida de Chivay, 3: Entre Achoma y Maca, 4: Cruz del Cóndor (Cabanoconde). 
amplia franja entre la zona abiótica del Desierto Pacífico (Galán de Mera et al., 1997) y los pajonaltolares meso-supratropicales seco-aridos del orden Parastrephietalia lepidophyllae (Navarro, 1993), y constituye la cabeza de serie del WeberbauerocereoCorryocacteto brevistyli $S$.

\section{Grindelio bolivianae-Corryocactetum puquiensis}

Galán de Mera y Gómez Carrión ass. nova

[Tabla 3, sintypus: inv. 2]

Vegetación dominada por Corryocactus puquiensis, que se asienta en laderas rocosas y desprendimientos con una pendiente del 50 al $90 \%$, donde abundan también matorrales y gramíneas frecuentes en el piso meso-supratropical semiáridoárido y plantas rupícolas (Notholaenetea niveae Gutte 1986: Cheilanthes pruinata, Notholaena nivea) y de canchales (Polypodio squamulosiTillandsietea usneoidis Bolòs, Cervi \& Hatschbach 1991: Puya ferruginea, Tillandsia usneoides). C. puquiensis es un endemismo de los valles profundos interandinos del departamento de Arequipa (Rauh, 1958), que da lugar a formaciones bastante densas junto a otras plantas suculentas, como Opuntia exaltata, O. soehrensii o Puya ferruginea. También son muy frecuentes los caméfitos esclerófilos, como Chersodoma jodoppapa, Grindelia boliviana o Proustia berberidifolia.

En los valles encajados del departamento de Arequipa, entre las poblaciones de Chivay y Cabanoconde, el abandono de los cultivos conlleva la instalación de esta asociación como comunidad basal, donde Corryocactus puquiensis se comporta como planta pionera y va acompañada de plantas nitrófilas de suelos removidos por la acción humana (Alonsoa acutifolia, Chenopodium petiolare, Ophryosporus peruvianus, Sarcostemma solanoides).

Fitotopográficamente, esta asociación contacta hacia el interior de los valles con la vegetación riparia del Cortaderion jubatae (Galán de Mera, 1995), y sobre los $3700 \mathrm{~m}$ con los pajonal-tolares meso-supratropicales de Parastrephia lepidophylla, constituyendo la cabeza de serie del Grindelio bolivianae-Corryocacteto puquiensis $S$.

\section{ESQUEMA SINTAXONÓMICO}

OPUNTIETEA SPHAERICAE Galán de Mera y Vicente Orellana 1996

+ Oreocereo leucotrichi-Neoraimondietalia arequipensis Galán de Mera y Vicente Orellana 1996

* Corryocaction brevistyli Galán de Mera y Vicente Orellana 1996

1. Corryocacto aurei-Browningietum candelaris Galán de Mera y Vicente Orellana 1996 [Comunidades mesotropicales hiperáridas del departamento de Tacna]

2. Oreocereo tacnaensis-Corryocactetum brevistyli Galán de Mera y Vicente Orellana 1996 [Comunidades mesotropicales árido-semiáridas del departamento de Tacna]

3. Weberbauerocereo weberbaueri-Corryocactetum brevistyli Galán de Mera y Gómez Carrión ass. nova [Cardonales mesotropicales semiárido-áridos del departamento de Arequipa]

4. Grindelio bolivianae-Corryocactetum puquiensis Galán de Mera y Gómez Carrión ass. nova [Cardonales meso-supratropicales semiáridoáridos de los valles interiores de Arequipa]

AGRADECIMIENTOS. Deseamos expresar nuestro agradecimiento a la Unidad de Postgrado de la Facultad de Biología de la Universidad Nacional Mayor de San Marcos (Lima, Perú) por las ayudas facilitadas durante la realización de este estudio tanto en el campo como en el herbario USM; también a la Agencia Española de Cooperación Internacional (AECI) en Lima, y muy especialmente al proyecto Araucaria-Valle del Colca por su apoyo. Este trabajo ha sido realizado gracias a una beca del Programa de Cooperación Interuniversitaria de la AECI.

\section{BIBLIOGRAFÍA}

ARAGÓN, G.A.-1980- Cactáceas de los alrededores de la ciudad de Arequipa. Bol. Lima (separata): 3-22.

ARÉVALO DEL CARPIO, C. - 1993- Visita a los alrededores de la ciudad de Arequipa y punta de Corio (Islay). Quepo 7: 46-55.

BARKMAN, J.J., J. MORAVEC \& S. RAUSCHERT -1986- Code der pflanzensoziologischen 
Nomenklatur. Vegetatio 67(3): 145-195.

BOLÒS, O., A.C. CERVI y G. HATSCHBACH 1991- Estudios sobre la vegetación del estado de Paraná (Brasil meridional). Collect. Bot. (Barcelona) 20: 79-182.

BRAKO, L. y J.L. ZARUCCHI -1993-Catálogo de las Angiospermas y Gimnospermas del Perú. Missouri Botanical Garden. St. Louis.

BRAUN-BLANQUET, J. -1932- Plant Sociology. The Study of Plant Communities. McGraw-Hill, New York and London.

GALÁN DE MERA, A. -1994- Sinopsis de las Pontederiáceas del Perú. Arnaldoa 2(2): 47-56.

GALÁN DE MERA, A. -1995- Ensayo sintaxonómico sobre las comunidades vegetales acuáticas del Perú. Arnaldoa 3(1): 51-58.

GALÁN DE MERA, A. -2000- La predicción climática de la vegetación. Una nueva perspectiva en agricultura. Anales Científicos XX RELAR. Instituto de Defensa del Medio Ambiente. Arequipa.

GALÁN DE MERA, A. y J.A. VICENTE ORELLANA -1996- Las comunidades con Corryocactus brevistylus del sur del Perú. Phytologia 80 (1): 40-47.

GALÁN DE MERA, A., J.A. VICENTE ORELLANA, J.A. LUCAS GARCÍA \& A. PROBANZA LOBO -1997- Phytogeographical sectoring of the Peruvian coast. Global Ecol. Biogeogr. Lett. 6: 349-367.

GUTTE, P. -1986- Beitrag zur Kenntnis zentralperuanischer Pflanzengesellschaften III. Pflanzengesellschaften der subalpinen Stufe. Feddes Repert. 97: 319-371.

IGM (Instituto de Geología y Minería) - 1975- Mapa geológico del Perú (1: 1.000.000). Lima.

LINARES, E. -2000- Diagnóstico de los recursos de flora y fauna de la Reserva Nacional de Salinas y Aguada Blanca. Tesis de Maestría inédita. Universidad Nacional de San Agustín. Arequipa.

NAVARRO, G. -1993- Vegetación de Bolivia: el Altiplano meridional. Rivasgodaya 7: 69-98.

NAVARRO, G. -1996- Catálogo ecológico preliminar de las cactáceas de Bolivia. Lazaroa 17: $33-84$.

RAUH, W. -1958- Beiträg zur Kenntnis der peruanischen Kakteenvegetation. Stizungsber. Heidelberger Akad. Wiss., Math.-Naturwiss.
RIVAS-MARTÍNEZ, S. -1987- Memoria del mapa de series de vegetación de España. ICONA. Madrid.

RIVAS-MARTÍNEZ, S. -1997- Syntaxonomical synopsis of the potential natural plant communities of North America, I. Itinera Geobotanica 10: 5-148.

STRAHLER, A.N. -1992- Geología Física. Omega. Barcelona.

TRYON, R. -1964- The Ferns of Peru. Polypodiaceae (Dennstaedtieae to Oleandreae). Contr. Gray Herb. 194: 3-253.

WEBERBAUER, A. - 1945-El mundo vegetal de los Andes peruanos. Ministerio de Agricultura. Lima.

Aceptado para su publicación en abril de 2001

Dirección de los autores. A. Galán de Mera: Laboratorio de Botánica, Universidad San PabloCEU, Apartado 67, E-28660- Boadilla del Monte, Madrid, E-mail: agalmer@ceu.es; J. Gómez Carrión: Museo de Historia Natural, Universidad Nacional Mayor de San Marcos, Apartado 140434, Lima 14, Perú. 